\title{
Enquête au Burkina Faso sur quelques projets de développement qu'intègrent les sciences sociales
}

Doris Bonnet

\section{(e) OpenEdition \\ Journals}

Édition électronique

URL : http://journals.openedition.org/apad/299

DOI : 10.4000/apad.299

ISSN : 1950-6929

Éditeur

LIT Verlag

Édition imprimée

Date de publication : 15 mars 1991

\section{Référence électronique}

Doris Bonnet, «Enquête au Burkina Faso sur quelques projets de développement qu'intègrent les sciences sociales », Bulletin de l'APAD [En ligne], 1 | 1991, mis en ligne le 23 juin 2006, consulté le 08 septembre 2020. URL : http://journals.openedition.org/apad/299; DOI : https://doi.org/10.4000/apad. 299

Ce document a été généré automatiquement le 8 septembre 2020.

Bulletin de l'APAD 


\title{
Enquête au Burkina Faso sur quelques projets de développement qu'intègrent les sciences sociales
}

\author{
Doris Bonnet
}

1 Le travail a été réalisé avec mon collègue burkinabé du CNRST, Oger Kaboré, auteur de la réflexion précédente sur les rapports entre recherche en sciences sociales et développement au Burkina Faso. Ma démarche, rapide, compte tenu du temps dont nous disposions, a été au contraire de rencontrer des opérateurs de développement afin d'inventorier quelques projets de développement qui comportent actuellement un volet "sciences sociales". Pour ce faire, je me suis adressée a la Mission française de coopération et d'action culturelle (MAC); la Caisse Centrale de Coopération Économique ; l'Agence Canadienne de Développement International ; au Ministère des Pays-Bas pour la Coopération au Développement.

2 En ce qui concerne la MAC, les renseignements communiqués par Nicole Giniès (Centre d'Information sur le Développement) ont permis d'identifier deux projets de développement en matière de santé qui avaient recours a des études ou des enquêtes anthropologiques, et un projet en matière de développement rural. Il s'agit des projets suivants :

3 - "Appui a la Santé Maternelle et Infantile en milieu urbain a Bobo Dioulasso" sous la responsabilité du Docteur Marie-Claire Dubois, qui, a la suite d'enquêtes épidémiologiques, a décidé de mener deux enquêtes sociologiques sur le système de santé dit traditionnel: les guérisseurs de la ville de Bobo Dioulasso spécialisés en pédiatrie et les divers itinéraires thérapeutiques des Bobolais. Le Docteur Dubois n'a pas eu recours a des professionnels mais a des étudiants burkinabé en sociologie (niveau maîtrise) qui en ont tiré un grand profit. Ils ont jugé ce travail très stimulant et leur permettant d'anticiper le type d'activités qu'ils pourraient mener ultérieurement. Néanmoins, la discipline a été essentiellement utilisée comme un outil méthodologique (l'enquête qualitative étant jugée complémentaire a l'enquête épidémiologique quantitative et dans une réflexion exclusivement médicale). 
4 - "Appui a la santé mentale au Burkina Faso", sous la responsabilité du Dr Gérard Mitelberg. Deux études ont été commanditées par le Dr Mitelberg. L'une portait sur les représentations de la maladie mentale et de l'épilepsie chez les Mossi du Burkina Faso réalisée par moi-même dans le cadre d'un accord avec l'ORSTOM; l'autre sur les représentations de la maladie mentale chez les Lobi (population présentant de grandes résistances au système de soins moderne) réalisée par Michèle Cros de l'université de Bordeaux. Ces études menées par des professionnels ont été réalisées sous forme de prestations de service. Elles n'ont pas véritablement engagé un travail d'équipe.

5 - "Formation technique continue dans cinq provinces de l'ouest Burkina", sous la responsabilité de Alain Demaison. A partir d'enquêtes de terrain de type ethno-linguistique (recueil et analyse des termes utilisés dans les activités agro-pastorales), des livrets didactiques ont été imprimés en langue vernaculaire et sont utilisés dans des Ateliers Techniques Villageois (ATV) afin de confronter au sein de réunions les discours et les méthodes en matière d'innovation. Les questionnaires ont été élaborés par un professionnel, Yannick Jaffré, ancien assistant technique en formation au Burkina Faso, psychologue de formation.

$6 \quad$ L'intervention des sciences sociales dans ces projets permet d'observer qu'elle dépend de la volonté du chef de projet plus que d'une politique générale de la MAC ou du Ministère français de la Coopération. Néanmoins, la politique de coopération "par projet" (et non plus de "substitution») permet que ce type de prestation soit dorénavant possible. La formation continue des assistants techniques assurée par le Ministère français de la Coopération devrait leur permettre de mieux comprendre ce qu'est le métier d'anthropologue, les limites épistémologiques de la discipline et en quoi elle peut leur être utile.

7 La Caisse Centrale de Coopération Économique a engagé au cours de l'année 1990, dans le cadre du projet national "Gestion des Terroirs Villageois", une série d'études socio-anthropologiques sur les trames foncières dans divers départements du Burkina Faso, travaux menés par des enseignants coopérants de l'université de Ouagadougou ( $R$. Brand, J. Bouju) ou des professionnels de divers corps d'origine (D. Kintz, B. Martinelli, A. Poloni). Il s'agit, pour la plupart d'entre elles, d'études de type "connaissance du milieu" comprenant un important recueil de données dans le but de mieux comprendre les mécanismes du pouvoir d'appropriation de la terre et du droit foncier ainsi que l'organisation et le fonctionnement des activités non agricoles.

8 Par ailleurs, les principes d'intervention de la Caisse centrale, au niveau du développement local, tendent a favoriser la mise en place d'équipes provinciales pluri-disciplinaires où les sociologues burkinabè peuvent trouver une fonction.

9 On constate donc, la aussi, une évolution favorable dans la prise en compte des modes d'organisation sociale traditionnels par rapport à l'implantation de nouveaux régimes fonciers et dans l'exploitation des ressources humaines locales.

10 La coopération canadienne utilise aussi, mais en avant-projet, des chercheurs en sciences sociales. Il s'agit surtout d'experts canadiens (avec des homologues burkinabé) en socio-économie ou en géographie. Une réflexion pluri-disciplinaire est menée au sein de chaque projet afin qu'à tout projet de développement social (alphabétisation, mise en place d'un comité de gestion) ainsi qu'un projet d'«appropriation du développement " (démarche pédagogique). Il semble que la demande vis-à-vis des 
sciences sociales consiste surtout à recueillir des données factuelles sur la zone où le projet va s'implanter.

11 Selon les représentants du ministère de la coopération des Pays-Bas, "la culture d'une société est la base et le point de départ du développement". Ainsi, les Hollandais n'ont pas hésité à nommer un anthropologue (S. Zanen) responsable d'un projet de développement rural dans le nord-est du pays ("Programmation et exécution du développement intégré") qui couvre les objectifs suivants: planification régionale, promotion agro-pastorale, auto-promotion paysanne, approvisionnement en eau potable, santé communautaire et éducation. Des recherches anthropologiques ont été menées, dans le cadre de ce projet sur le régime agraire et foncier (par S. Luning, anthropologue hollandaise) ainsi que sur les conceptions de la maladie et sur la malnutrition chez l'enfant (par une étudiante en médecine des Pays-Bas). Un projet de recherche sur l'auto-promotion paysanne et sur la notion de communauté doit être effectué par J. Till, anthropologue hollandais.

Ces études ont "intérêt de ne pas être uniquement des études de milieu. Elles sont reprises par le coordinateur du projet dans le but "d'intégrer les aspects culturels dans le développement" et afin d'élaborer le projet en "respectant l'environnement socio-culture".

Par manque de temps, nous n'avons pas poursuivi comme nous l'aurions souhaité ce type d'investigation, par exemple auprès de la coopération allemande ou italienne.

Mais, d'ores et déjà, on peut remarquer une prise en compte de plus en plus importante des sciences sociales dans les projets de développement même si elle repose parfois sur la personnalité des chefs de projet, ceux-ci étant plus ou moins réceptifs à ce type d'intervention. Ceci dit, on peut aussi souligner que les structures administratives ne s'opposent pas aux choix des chefs de projet, ce qui témoigne d'une évolution positive des bailleurs de fonds vis -à -vis des sciences sociales.

Néanmoins on a pu observer, au cours de cette brève enquête, que les sciences sociales sont souvent utilisées uniquement comme des outils méthodologiques ou comme des recueils d'information de type "connaissance du milieu". On ne leur donne pas véritablement l'ambition de réfléchir sur le développement ou le changement social en soi.

Enfin, on a fait remarquer plus haut que le travail mené par des étudiants ou des professionnels n'avaient pas conduit jusqu'à aujourd'hui à la mise en place d'équipes pluri-disciplinaires. Les interventions restent sporadiques et "au service" d'une autre discipline. Elles témoignent pourtant de la reconnaissance des conceptions et des pratiques locales par les opérateurs du développement et de la nécessité de leur compréhension pour mieux maitriser les difficultés d'application d'un projet.

Que soient remerciés ici : N. Giniès (Centre d'Information sur le Développement), Dr. M.-C. Dubois (Ministère français de la Coopération et du Développement), Dr. G. Mitelberg (Ministère français de la Coopération et du Développement), A. Demaison (Ministère français de la Coopération et du Développement), S. de Pas (Caisse Centrale de Coopération Économique), D. de la Croix (Caisse Centrale de Coopération Économique), S. Moreau (Agence Canadienne du Développement), A. Gosses (Ministère des Pays-Bas pour la Coopération au Développement), S. Zanen (Programmation et exécution du développement intégré, Kaya). 